\title{
Automated smart hydroponics system using internet of things
}

\author{
Ravi Lakshmanan, Mohamed Djama, Sathish Kumar Selvaperumal, Raed Abdulla \\ Faculty of Computing, Engineering and Technology, Asia Pacific University of Technology and Innovation (APU), \\ Technology Park Malaysia, Malaysia
}

\begin{tabular}{l}
\hline \hline Article Info \\
\hline Article history: \\
Received Feb 22, 2020 \\
Revised May 27, 2020 \\
Accepted Jun 17, 2020 \\
\hline
\end{tabular}

Keywords:

Horticultural lighting Internet of things

MQTT

NFT

PCB

Smart hydroponics system

\begin{abstract}
This paper presents a design and implementation of an automated smart hydroponics system using internet of things. The challenges to be solved with this system are the increasing food demand in the world, the need of market of new sustainable method of farming using the Internet of Things. The design was implemented using NodeMcu, Node Red, MQTT and sensors that were chosen during component selection based on required parameters and sending it to the cloud to monitor and be processed. Investigation on previous works done and a review of Internet of Things and Hydroponic systems were done. First the prototype was constructed, programmed and tested, as well as sensors data between two different environments were taken and monitored on cloud-based web page with mobile application. Moreover, a bot has been introduced to control the supply chain and for notification purposes. The system improved its performance and allows it to successfully achieve the aim of the entire system implemented. There are some limitations which can be improved as future work such as including data science with the usage of the artificial intelligence to further improve the crops and get better outcome. Lastly to design end user platform to ease user interaction by using attractive design with no technical configuration involved.
\end{abstract}

Copyright $@ 2020$ Institute of Advanced Engineering and Science. All rights reserved.

\section{Corresponding Author:}

Raed Abdulla,

School of Engineering,

Asia Pacific University of Technology and Innovation (APU),

Technology Park Malaysia, Bukit Jalil, Kuala Lumpur 57000, Malaysia.

Email: dr.raed@apu.edu.my

\section{INTRODUCTION}

Agriculture is the cornerstone of human civilization and is responsible for the production of quality food for the human population [1-4]. To ensure the availability of inexpensive and quality products to meet the increasing food demand, intensification of the growing practices is necessary. By definition, hydroponics is subset of hydro culture, the method of growing plants without the use of soil, using mineral nutrient solutions in a water solvent [5-9]. As defined, plants in hydroponics system are grown without the use of soil. Plants receive all the essential nutrients from a nutrient rich water-based solution, whereby the water is mixed with the specified solutions for the plant to be grown. Therefore, there are a variety of hydroponic methods or technique in which plants can grow in a non-soil medium or directly in the nutrient rich water-based solution. These operations are controlled systematically and therefore tend to produce higher than traditional farming. For the past couple of years this method has been implemented and used in urban areas to improve the access to a fresh food $[10,11]$. The Internet of Things is an emerging topic of technical, social, and economic significance [12]. Consumer products, durable goods, cars and trucks, industrial and utility components, sensors, and other everyday objects are being combined with Internet connectivity and powerful data analytic capabilities that promise to transform the way we work, live, and play [13]. 
Projections for the impact of IOT on the Internet and economy are impressive, with some anticipating as many as 100 billion connected IOT devices and a global economic impact of more than $\$ 11$ trillion by 2025 [14]. Automation has changed the way we work, live and make things. Moreover, there are a lot of challenges in maintaining automated products, which lies under the high cost and upgrading those products will mostly cost high prices depending on the product and the manufacturer. Therefore, IOT which is an emerging technology might solve some of those problems [15]. A few works have reported a methodology on how to transfer the data from one node to another. A system that transfers the data using ZigBee from sensor node has been proposed by [16-22], thus a raspberry pi connected with ZigBee module receives the data from the sensor node and passes it to the PC node whereby data is received and processed. Another researcher proposed a much better system, [23] that uses four type of sensors to detect rainfall, temperature, moisture and light then sends to Thingspeak which is a paid cloud that receives data via MQTT protocol. Commonly most of the researchers have proposed systems that utilizes ZigBee as communication medium and without any cloud infrastructure to analyze and process data and control actuators in real time [24]. Researchers implemented hydroponics using electronic circuit, nutrients and water to be automatically monitored which led to higher productivity. Implemented pest detection and to connect Wi-Fi module in order to automate the system to be more flexible and yielded to save water and fertilizers [25-31].

There many reasons that push us to go for hydroponics, starting with the global food demand which is increasing rapidly and expected to be anywhere between $59 \%$ to $98 \%$ by 2050 . This will shape agriculture market a put a lot of pressure in ways we have never seen before, whereby farmers worldwide will have to increase their crop production, either by enhancing and improving the productivity of the existing agriculture land by fertilizing and irrigation or by increasing the amount of agricultural land. Secondly, water conservation which an increasing concern in the society. If we look at the average soil gardener usually every few days, they use a good amount of water into their soil, ensuring good penetration into the soil so the plants roots can make the most out of it. Thirdly less pets and diseases and higher productivity which means healthier than the traditional farming.

The proposed system here is an automated smart hydroponics system, whereby this system will be able to add and implement Internet of Things concept and functionality to the current existing hydroponics system. Moreover, with the use of Internet of things the system can be monitored and controlled from anywhere via the internet, therefore parameters such as water, $\mathrm{pH}$ level, room temperature, nutrient rich water-based solution temperature and room humidity parameters can be viewed in real time. One of the key features of this system is the ability to make decision and send command from a friendly user interface based on the shown real time parameters.

\section{PROPOSED DESIGN METHODOLOGY}

Figure 1 illustrates the main flowchart of the proposed system and it consists of three major sections which are the client side, server side and graphical user interface.

\subsection{Client side}

In order to monitor real time parameters, the client side must setup accordingly, therefore three stages must be considered. Programming the microcontroller with sensor and actuators, include the initiating section whereby hydroponics has been analyzed accordingly to obtain the required sensor to measure the different parameters such as the $\mathrm{pH}$ level, Humidity and temperature of the room, temperature level when water is mixed with the solution and the horticultural lighting to provide the right illumination fo the plant at night. Followed up with the electrical wiring for the system and PCB designing whereby after the programming the sensors and the microcontroller together, all the wiring and power supply are assembled as one part with $5 \mathrm{~V}$, moreover the used technique for the hydroponics is Nutrient Film Technique.

\subsection{Server side}

The second stage of the system is to build the server side of the system. This mainly consists of two parts, setting the MQTT broker server which is used to transfer the data from microcontroller to any platform that can read MQTT data. Initially this was done to secure the data and enhance the efficiency and the reliability of the data sent and received from and to the microcontroller from any possible third party to access to the data. In order to fully deploy the MQTT broker server, host was required therefore a raspberry pi 3 has been used as the host for the server at port 1883 to access the mosquito MQTT broker server authentication (username and password). This is done to ensure only appropriate devices can be connected and read and write throughout the server. The second part of the second stage is the setup of the Internet of Things platform. Many platforms are available and provide a wide variety of IOT services, but due to the price that will be required with the time and according to one of the objectives which was to reduce 
the cost of the system, a free IOT server has been deployed called NodeRed. According to NodeRed Org, (2016) NodeRed is a visual tool for wiring the Internet of Things. Node-Red is a tool for wiring together hardware devices, APIs and online services in new and interesting ways. Hence after deploying the NodeRed server, authenticating is done to secure the access to the server and password is encrypted to ensure the maximum amount of security. Moreover, the second stage servers will require hosting to provide accessibility from anywhere around the world therefore a hosting is required. For this system a dynamic domain name will be used to reroute the traffics to selected domain name from the raspberry pi host.

\subsection{Graphical user interface}

The last stage of the system is building a friendly graphical user interface to display the real time data; therefore, two proposed methodologies will be discussed here. The first method is to build a graphical user interface with the NodeRed itself which displays the data in web form that could be hosted in the cloud where an IOS mobile app will be built based on that Graphical user interface to provide ease and accessibility for the system across the different platforms. The second stage is optional which will require building entire IOT server from the scratch using HTML, CSS and java script and host it in a private hosting, which will be done if the time and resources allows.

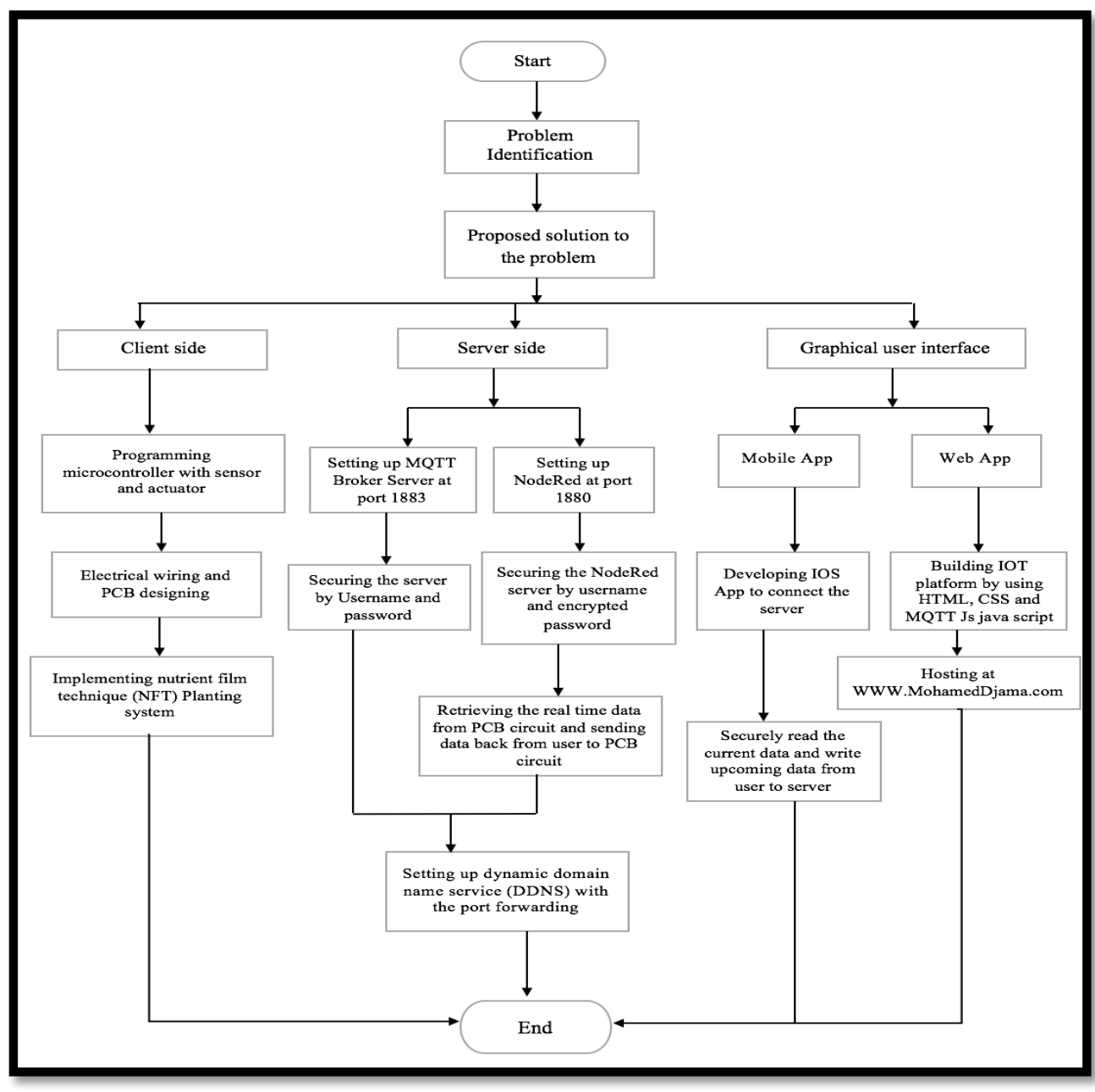

Figure 1. Proposed automated smart hydroponics system flow chart

\section{SYSTEM IMPEMENTATION}

\subsection{Overall block diagram}

The overall block diagram shows the main parts and components of the system and the way of communications between the sensors, relays, PCB, Node-Red, GUI, and the mobile application. The overall block diagram shown in Figure 2 shows the main parts of the system and the communication medium 
between the software and the hardware components. The system works based on the data acquired by the sensors or based on the commands given by the user. There are 4 sensors which are water, temperature sensor, $\mathrm{pH}$ level sensor, and the room humidity and temperature sensor. These sensors are connected to the PCB where the data acquired by the sensors will be transferred through the internet via the MQTT broker and then to the Node-red where the data will be analyzed and based on that the action will be taken automatically.

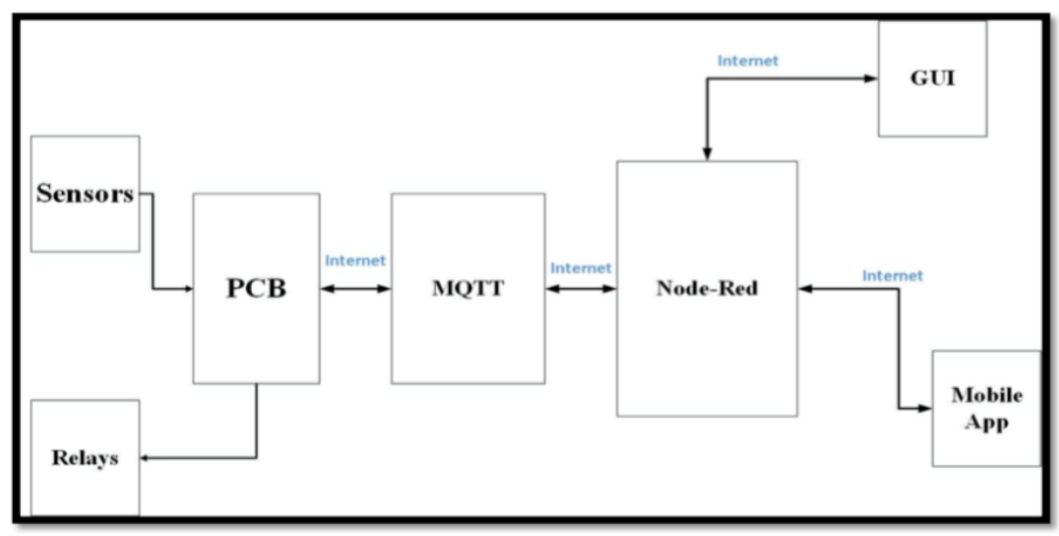

Figure 2. Overall block diagram of automated smart hydroponics system

The GUI and the mobile app of the system shows the system status and all the related data acquired by the sensors where the user can take action and send commands based on that or based on what is needed. Software used in this project to design and construct the system before implementation is explained further. Starting with PCB, Design Spark software has been used to design the two layers PCB design after initial hand sketch. Figure 3 shows the power layer, whereby only node connections are directly linked with MIC29302WU1543P that solves the power and manage it accordingly between the microcontrollers, relays, analogue and digital nodes. Figure 4 illustrates the digital, analogue, I2C and PWM wiring between the microcontroller NodeMcu Esp8266 and the end nodes.

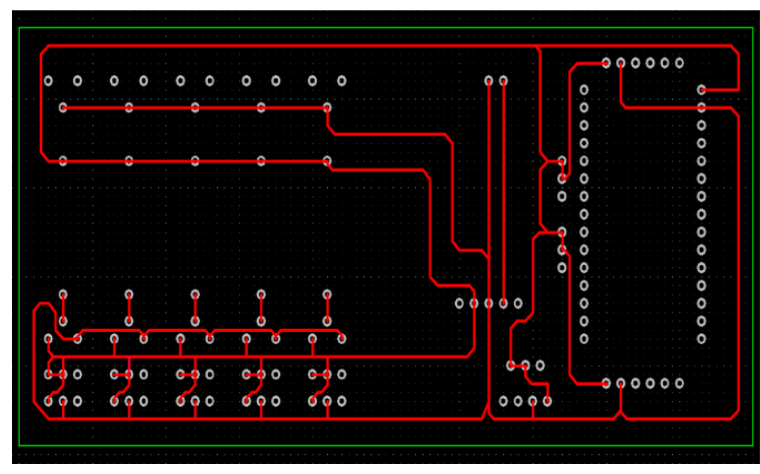

Figure 3. Power layer design for the PCB

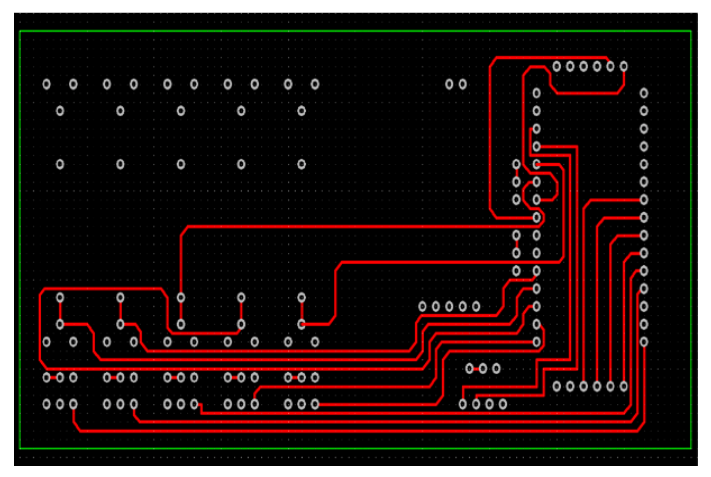

Figure 4. Analogue \& digital layer design for the PCB

Moreover, ultrasonic sensors with a NodeMcu Esp8266 are designed separately due to the functionality of that node. An Ultrasonic sensor is a device that can measure the distance to an object by using sound waves. It measures distance by sending out a sound wave at a specific frequency and listening for that sound wave to bounce back from the object or obstacle by using mathematical formula. The NodeMcu provide only $3.3 \mathrm{~V}$ therefore an alternative solution has been developed. By default, the NodeMcu input voltage is $5 \mathrm{~V}$ and gets the input in two ways, either via a USB or Vin pin. Assuming a Y shape whereby the input voltage is expected to come from the USB port, hence it's expected to have $5 \mathrm{~V}$ in the Vin pin which is used in the circuit as shown in Figure 5. 


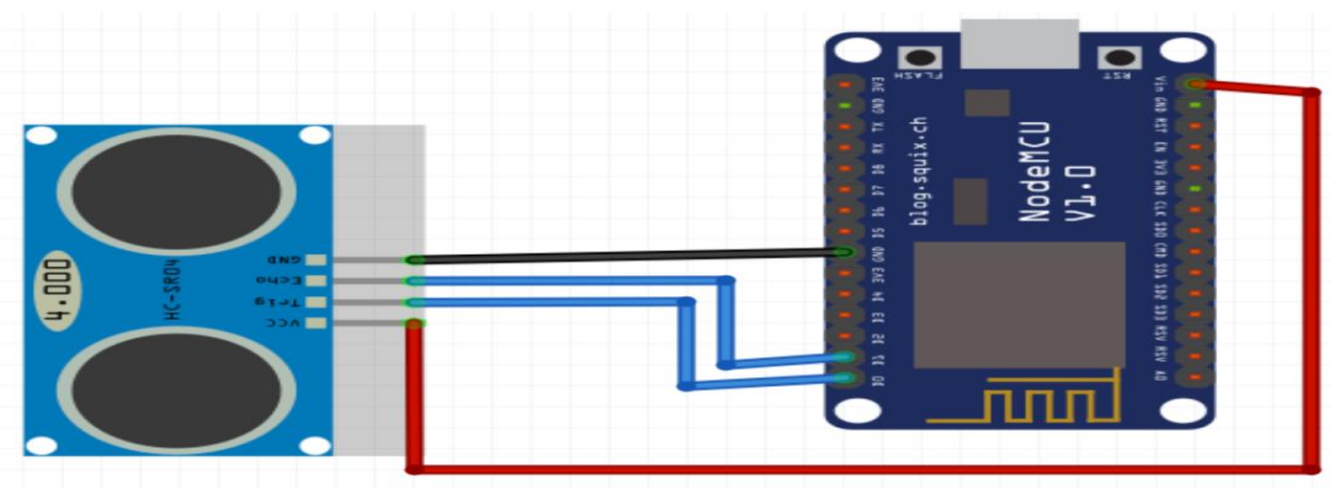

Figure 5. NodeMcu with ultrasonic sensor for stock chain

Additionally, the overall structure of the proposed automated smart hydroponics system has been sketched and drawn with Solid Work before building the structure or the prototype. Figure 6 illustrates the 3D model presented from different views. Starting with the top view with the actual length and width of the pipes and lights. Moreover, enclosure box has been added for the PCB for many reasons, mainly to avoid any short circuit for the PCB that leads to failure and burn the entire circuit. In addition to safety of the users, the Omron relay is designed to control $220 \mathrm{~V}$ horticultural light used in the hydroponics system to give the plant what they need from the sun. As the incoming $220 \mathrm{~V}$ enters the circuit there is a higher risk of electrical shock to the end user that can lead to a disastrous outcome, thus the enclosure was needed. As shown in Figure 7 the Solid Work 3D design for the box, with the use of 3D printer, the design was saved in. STL format and sliced with cura-engine which converts the 3D design into printing structures for the $3 \mathrm{D}$ printer.

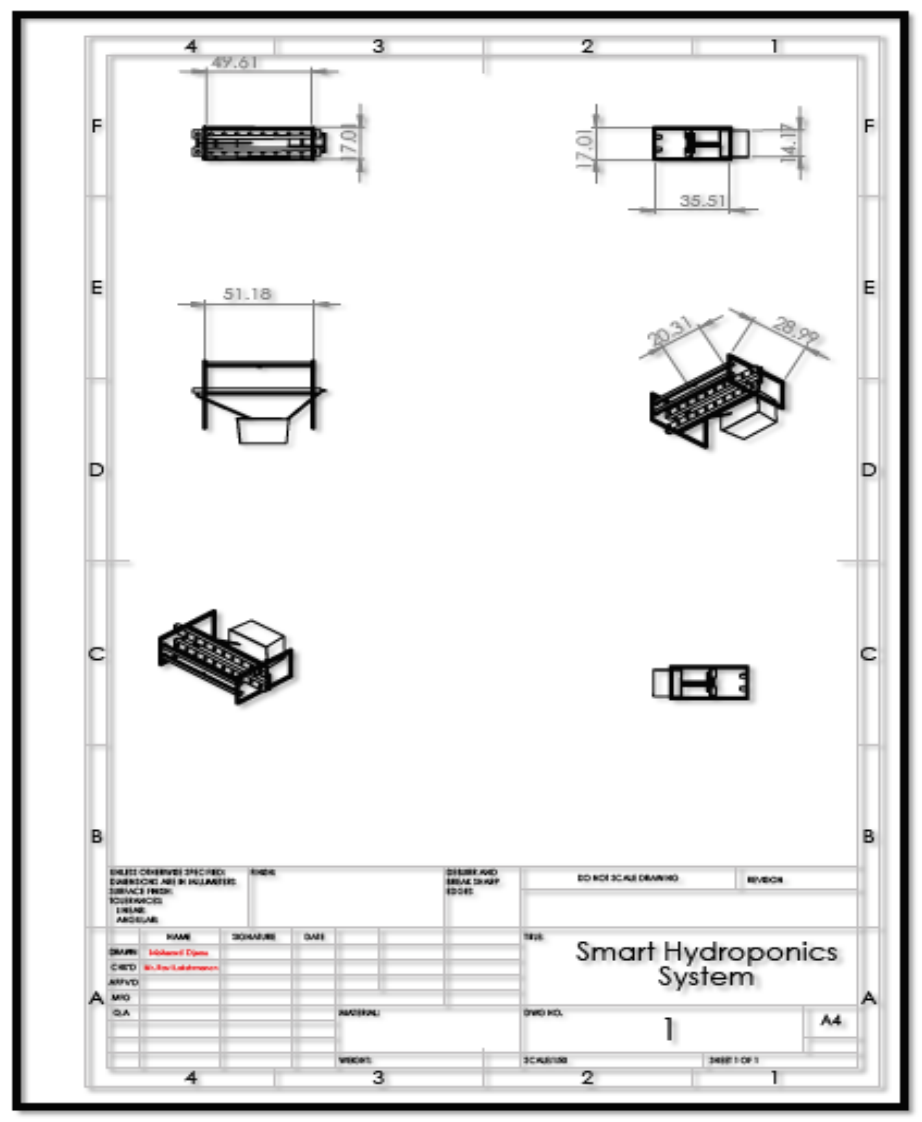

Figure 6. Automated smart hydroponics prototype design 


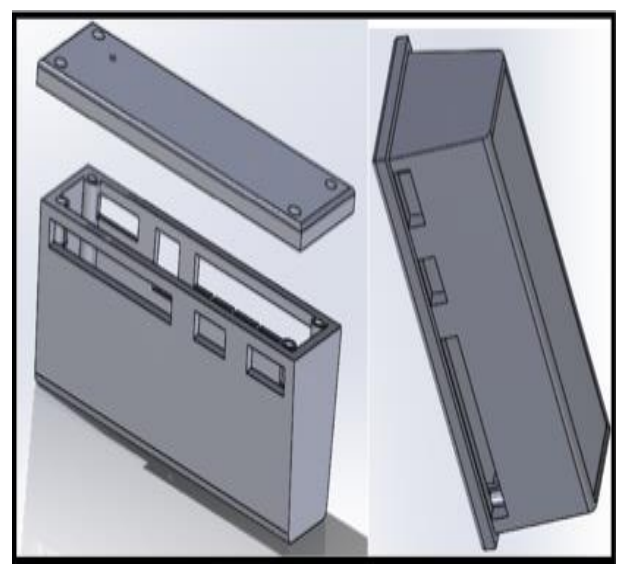

Figure 7. PCB enclosure box

\subsection{Working principle}

The working principle of the system implemented is explained with the help of the structure shown in Figure 8. The smart hydroponics system which consists of three major parts Input data, Cloud server (Monitoring and controlling) and Output data. Starting with the input data, as IOT stands for the internet of things, basically a group of things more specifically the sensors communicate with each other via a network, thus a group of sensors is required to make up the IOT system. The smart hydroponics system consists of variety of sensor that acquires the following data: room temperature, room humidity, water temperature and $\mathrm{pH}$ level and fertilizers level. In addition, the horticultural light status to be utilized for controlling purposes, with the telegram bot input data which is also considered as an input to the system for processing later on.

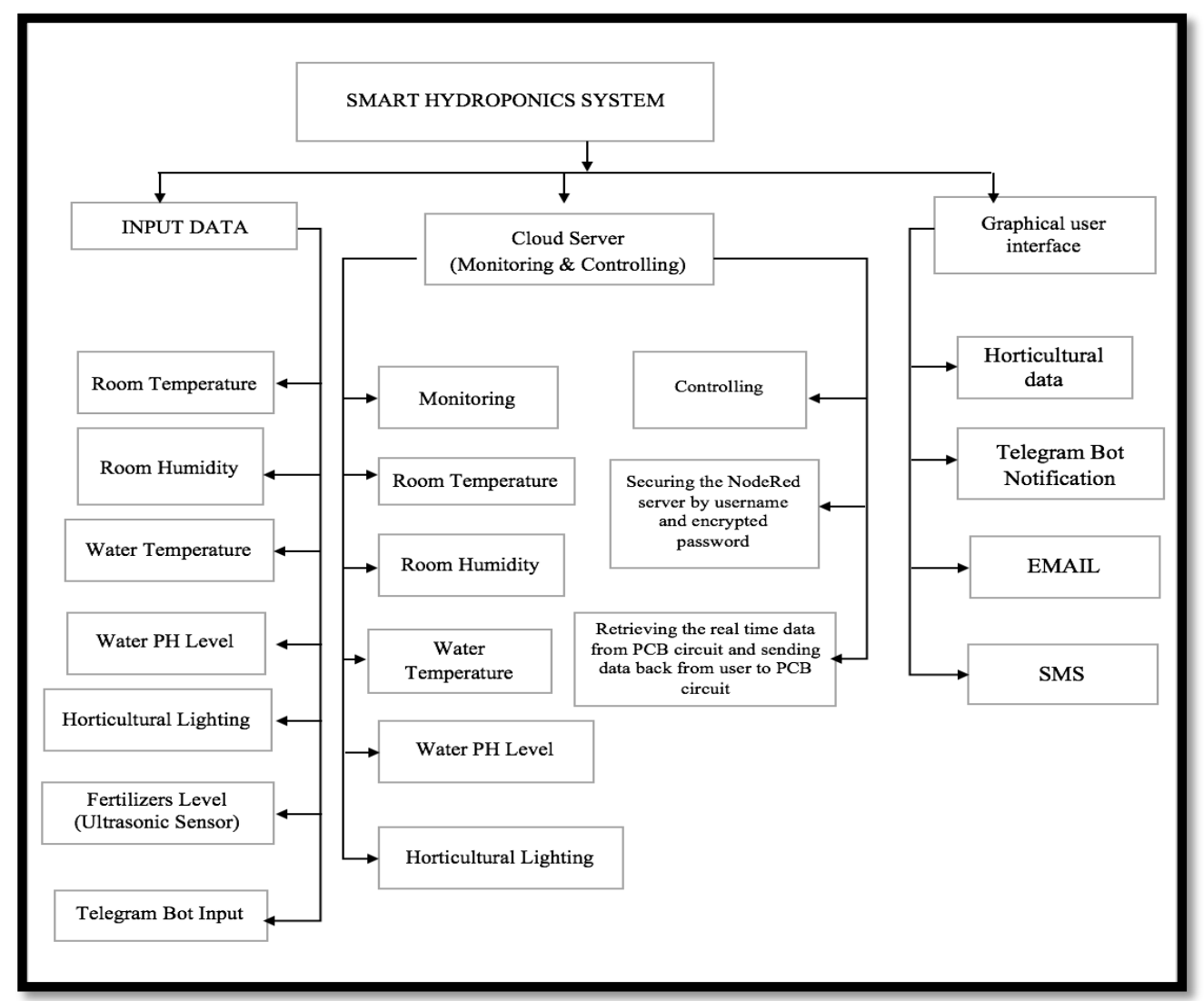

Figure 8. Automated smart hydroponics system structure 
By definition the internet of things is the ability to monitor and visualize data in real time and at the same time being able to send back data and control parameters over a network. To achieve that process an MQTT which stands for MQ Telemetry Transport is designed for low bandwidth high latency networks, a lightweight messaging protocol to publish and subscribe for topics. Moving on with cloud server a NodeRed server is a programming tool for wiring together hardware devices, APIs and online services in new and simplified ways. The cloud server section will be divided into two major part monitoring and controlling, monitoring will be done via a graphical user interface developed and configured from the NodeRed itself to display data like room temperature and humidity, water temperature and $\mathrm{pH}$ level and the status of the horticultural whether it's on or off in real time. For the controlling part of the platform API's like telegram and Nexmo for SMS are used to control and notify the end user or the owner for the status or any sort notification related to the system. The GUI buttons are used to control the horticultural light by turning it on or off via sending it to the appropriate MQTT server. Last but not least, after changes or modifications, the monitoring data updates it and notification are sent as an output to the system via notification to the telegram bot and SMS in case the end user isn't connected to the internet on his/her phone. Moreover, an email will be sent to the supplier before two-week time as a notification to bring the supply and a notification goes to the end user to get ready for the payment within two- week time too.

\section{HARDWARE AND SIMULATION RESULTS}

In this section, the results of hardware and software will be discussed, primarily the automated smart hydroponics system has been successfully deployed with Nutrient Film technique to feed the plants as shown in the Figure 9 where the plants are well grown. Eventually different plants with different seeds has been tested and has successfully grown up these plants. With the use of ultrasonic sensor, the automated smart hydroponics stock supply chain has been established and the result was fantastic. An email has been received and the SMS too, as shown in Figure 10, the email content received, the SMS and the telegram received by the owner of the system are illustrated. The IOS mobile application is really important for the project and hence the screenshot of the same is shown in Figure 11. It is successfully working and monitoring of the data and the ability to control it are shown in Figure 11. In fact, it's similar to the graphical user interface of the NodeRed server which provide authentication at the beginning and dynamic refresh with data integration as again shown in Figure 11.

The performance of the implemented automated smart hydroponics system was evaluated by conducting tests. Figures 12 and 13 illustrates the graph draw between different parameters such as water temperature, room temperature, room humidity and $\mathrm{pH}$, whose readings are taken from the sensors in two different environments, namely, at home and at the Asia Pacific University (APU).

Figure 14 shows the latency test of the MQTT broker servers in milliseconds (ms) with number of published message or the received throughput per seconds. Moreover, Mosquitto MQTT is chosen due to the consistency and the QOS (Quality of Service) in addition to the security provided for the server accessibility at port 1883. Thus, throughout this project Mosquitto is used to send data from NodeMcu ESP8266 to the cloud (NodeRed) and vice versa via established secure MQTT server. As shown in Figure 11, the sensor hub is connected to the $\mathrm{WiFi}$, so that the sensor data are received in the cloud.

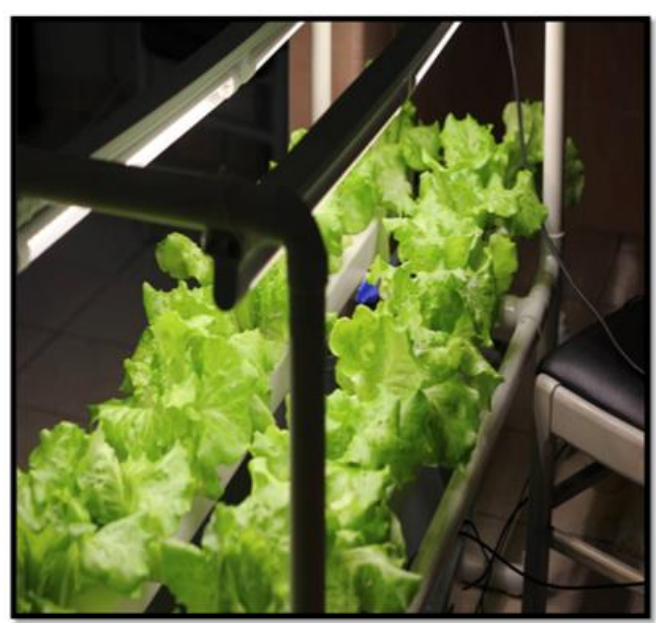

Figure 9. Smart hydroponics system used to grow a lettuce
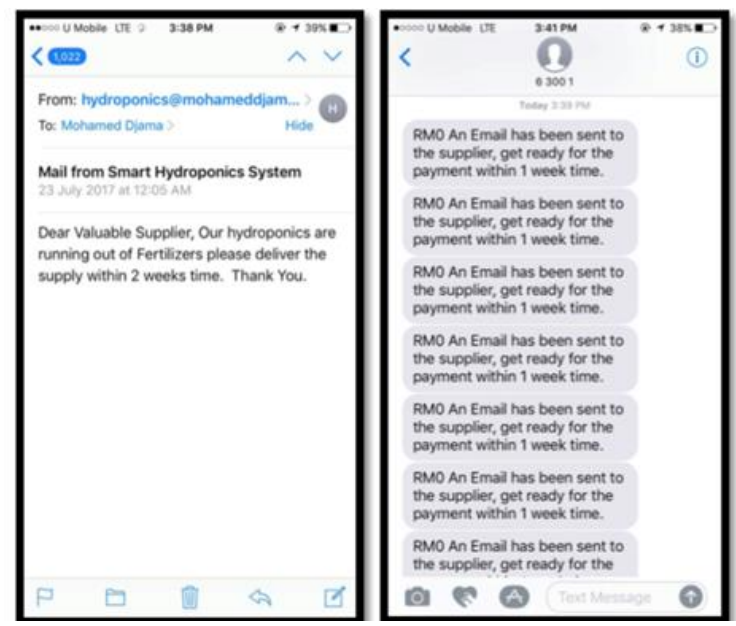

Figure 10. Smart hydroponics system supplier and owner notification 


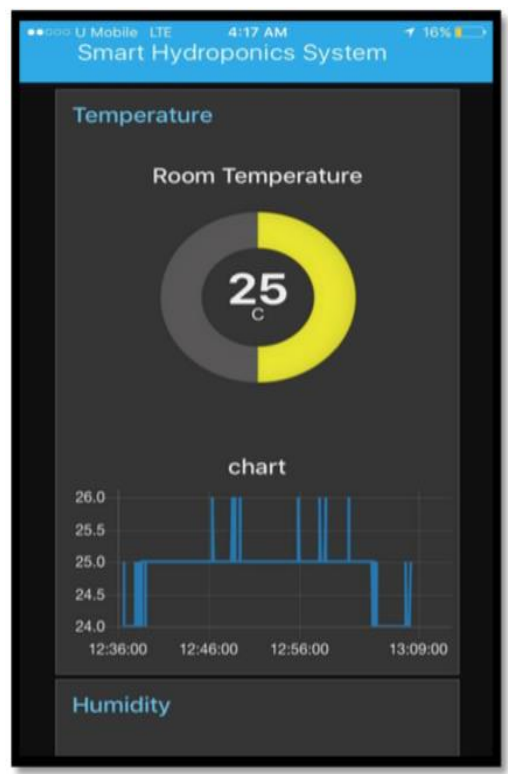

Figure 11. Automated smart hydroponics system IOS mobile application

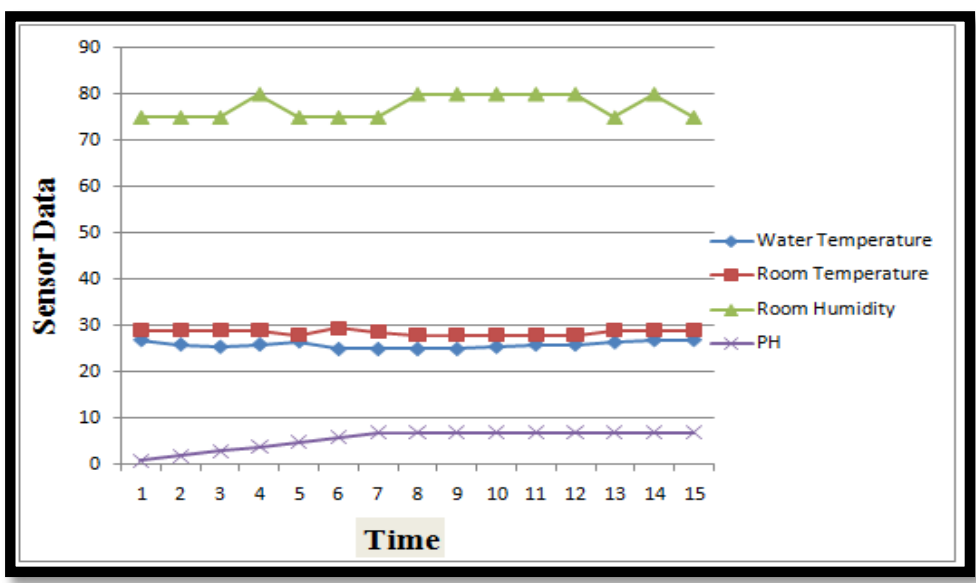

Figure 12. Sensor data collected from home

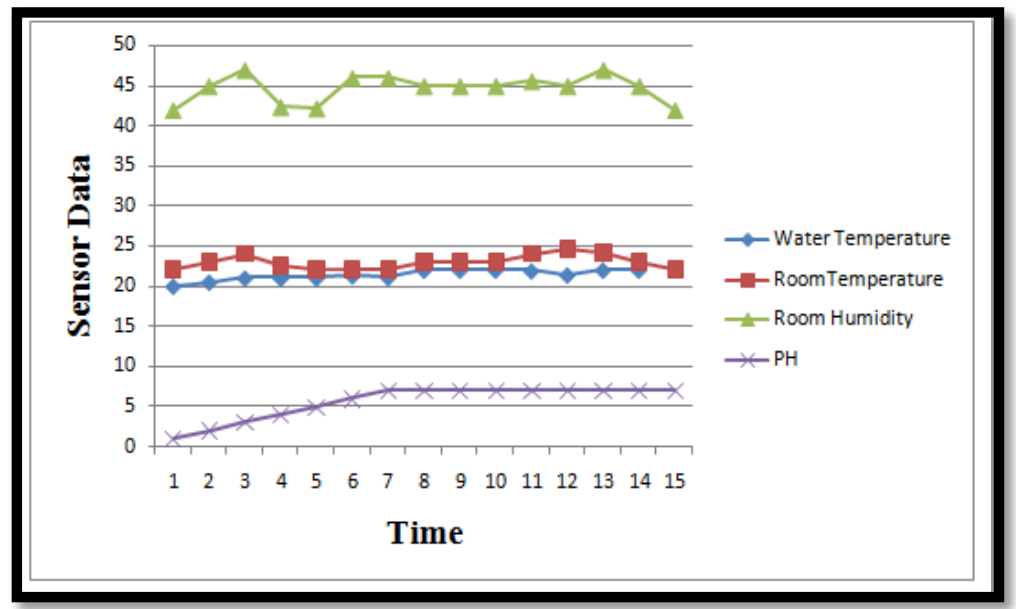

Figure 13. Sensor data collected from APU 


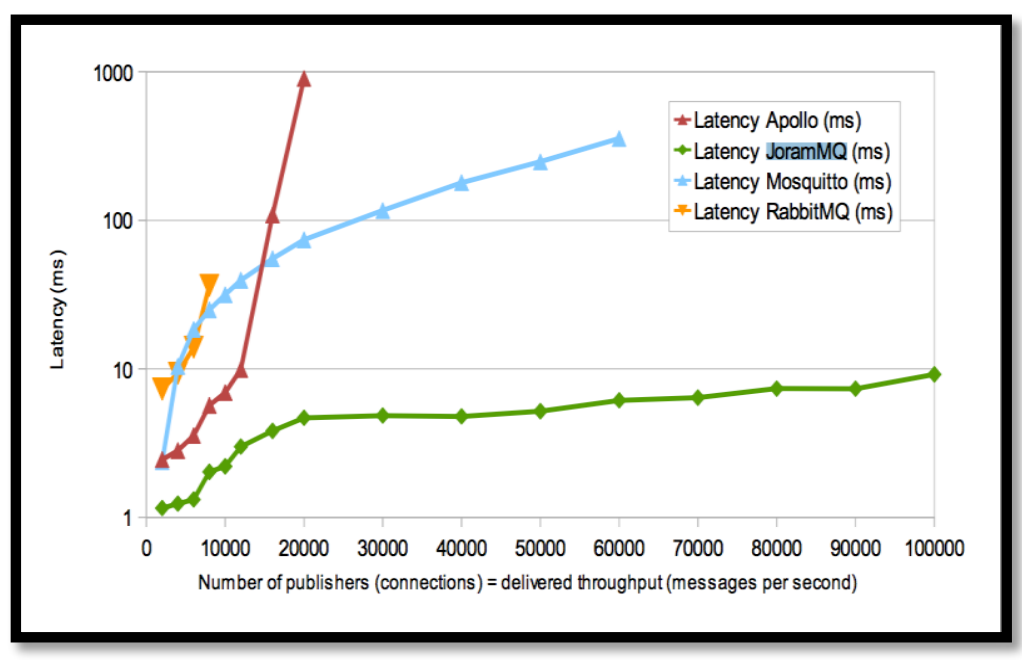

Figure 14. Latency test

\section{CONCLUSION}

The proposed system in this paper is an automated smart hydroponics system, whereby this system will be able to add and implement Internet of Things concept and functionality to the current existing hydroponics system. A GUI that allows the user to control and monitor real time data has been successfully achieved by developing a user interface node for the NodeRed and linked with the real-time sensor data. Moreover, it is hosted on the cloud at Nodered.MohamedDjama.com to make accessible from everywhere. Nginx server was used to forward traffics from port 1880 to 80 which HTTP but to add more security an SSL certificate has been issued and added therefore traffics are redirected to 443 which is HTTPS. Internet of things concept is implemented to the system to ease the process of growing food hydroponically by easing the process of growing and monitoring via secure cloud. By adding the internet of things, it solves one the key challenges in automation today which is maintenance, by providing a platform for monitoring the entire system from cloud, thus reducing the cost of maintenance by fraction. Lastly, the performance of the smart hydroponic system is evaluated. The automated smart hydroponics system is designed for indoor usage, for a bigger scale using Wi-Fi isn't the ideal wireless network, and the system is a success and implemented successfully. However, future work that can be done for further improvement will be by Integrating Data Analytics to the system or machine learning to develop algorithms to predict outcomes, add more sensors to get more accurate data and help the Artificial Intelligence system to predict the outcome better and by using intel $\mathrm{C} 1000$ microprocessor which comes built in with image processing for the crops health.

\section{REFERENCES}

[1] C. J. G. Aliac and E. Maravillas, "IOT Hydroponics Management System," in IEEE $10^{\text {th }}$ International Conference on Humanoid, Nanotechnology, Infromation Technology, Communication and Control, Environment and Management, Philipines, pp. 1-5, 2018.

[2] S. Navulur, et al., "Agricultural management through wireless sensors and internet of things," International Journal of Electrical and Computer Engineering (IJECE), vol. 7, no. 6, pp. 3492-3499, 2017.

[3] H. J. Dong, et al., "Interactive on smart classroom system using beacon technology," International Journal of Electrical and Computer Engineering (IJECE), vol. 9, no. 5, pp. 4250-4257, 2019.

[4] S. Padmadevi and V. Deepigha, "Smart E-Agriculture Monitoring Using Internet of Things," in Sixth International Conference on Emerging trends in Engineering and Technology (ICETET'16), Levengipuram, pp. 47-50, 2016.

[5] S. Keerthana, et al., "Automating and Analysing Greenhouse Hydroponic Farms using IOT," Interntaional Journal for Research in Applied Science \& Engineering Technology, vol. 6, no. 3, pp. 3325-3329, 2018.

[6] A. Rajeswari and S. Saipriya, "A survey on Hydroponic methods of smart farming and its effectiveness in reducing pesticide usage," International Journal of Pure and Applied Mathematics, vol. 119, no. 12, pp. 1503-1510, 2018.

[7] M. D. Sardare and S. V. Admane, "A Review on Plant without Soil Hydroponics," International Journal of Research in Engineering and Technology, vol. 2, no. 3, pp. 299-304, 2013.

[8] X. Wang and N. Liu, "The application of Internet of things in agricultural means of production supply chain management," Journal of Chemical and Pharmaceutical Research, vol. 6, no. 7, pp. 2304-2310, 2014.

[9] N. Vijayakumar and R. Ramya, "The real time monitoring of water quality in IoT environment," in Proceedings of International Conference on Innovations in Information, Embedded and Communication Systems, pp. 1-5, 2015. 
[10] N. Tanke, et al., "Automation of Hydroponic Installations using a Robot with position based visual feedback," Proceedings of the International Conference of Agricultural Engineering, pp. 1-6, 2012.

[11] I. Mohanraj, et al., "Field Monitoring and Automation using IOT in Agriculture Domain," Procedia Computer Science, vol. 93, pp. 931-939, 2016.

[12] J. Nuvvula, et al., "Environmental smart agriculture monitoring system using internet of Things," International Journal of Pure and Applied Mathematics, vol. 115, no. 6, pp. 313-320, 2017.

[13] D. Vyas, et al., "Smart Agriculture Monitoring and Data Acqusition System," International Research Journal of Engineering and Technology, vol. 3, no. 3, pp. 1823-1826, 2016.

[14] K. Rose, et al., "The Internet of Things and Overview," The Internet Society (ISOC), 2015.

[15] P. Sihombing, et al., "Automated Hydropincs nutrition plants systes using Arduino uno microcontroller based an android," Journal of Physics: Conference series, vol. 978, pp. 1-6, 2018.

[16] N. Gondchawar and R. S. Kawitkar, "IOT based smart agriculture," International Journal of Advanced Research in Computer and Communication Engineering, vol. 5, no. 6, pp. 838-842, 2016.

[17] A. Bagha and V. Madesetti, "Internet of Things: A Hands-on Approach," 2015. [Online]. Available: www.internet-of-things-book.com.

[18] S. S. Bedekar and M. A. Mechkul, "IOT Based Automated Irrigation System," International Journal of Modern Trends in Engineering and Research, vol. 2, no. 7, pp. 1532-1538, 2015.

[19] T Eldemerdash, et al., "IoT Based Smart Helmet for Mining Industry Application," International Journal of Advanced Science and Technology, vol. 29, no. 1, pp. 373-387, 2020.

[20] M. V. Shewale and D. S. Chaudhari, "IoT based plant Monitoring System for Hydroponics Agriculture: a Review," International Journal for Research in Applied Science \& Engineering Technology, vol. 6, no. 11, pp. 1628-1631, 2018.

[21] R. Rajkumar and R. Dharmaraj, "A Novel approach for smart Hydroponic Farming using IoT,” International Journal of Engineering Research in Computer Science and Engineering, vol. 5, no. 5, pp. 18-23, 2018.

[22] D. K. Sreekantha and A. M. Kavya, "Agricultural Crop Monitoring using IoT- A Study," in $201711^{\text {th }}$ International Conference on Intelligent Systems and Control (ISCO), pp. 134-139, 2017.

[23] S. A. Jaishetty and R. Patil, "IOT sensor network based approach for agricultural field monitoring and control," International Journal of Research in Engineering and Technology, vol. 5, no. 6, pp. 45-48, 2016.

[24] T. Bangera, et al., "IOT based Smart Village," International Journal of Engineering Trends and Technology, vol. 32, no. 6, pp. 301-305, 2016.

[25] S. Tembe, et al., "IoT based Automated Hydroponics System," International Journal of Scientific \& Engineering Research, vol. 9, no. 2, pp. 67-71, 2018.

[26] W. Lee and P. Law, "A case study in applying security design patterns for IoT software system," in 2017 International Conference on Applied System Innovation (ICASI), India, pp. 6-9, 2017.

[27] Charumathi S., et al., "Optimization and Control of Hydroponics Agriculture using IOT," Asian Journal of Applied Science and Technology, vol. 1, no. 2, pp. 96-98, 2017.

[28] P. P. Ray, "Internet of things for smart agriculture: Technologies, practices, and future direction," Journal of Ambient Intelligence and Smart Environments, vol. 9, pp. 395-420, 2017.

[29] F. Tongke, "Smart agriculture based on cloud computing and IOT," Journal of Convergence Information Technology, vol. 8, no. 2, pp. 1-7, 2013

[30] O. Mirabella and M. Brischetto, "A hybrid wired/wireless networking infrastructure for greenhouse management," IEEE Transaction on Instrumentation and Measurement, vol. 60, no. 2, pp. 398-407, 2011.

[31] P. Corke, et al., "Environmental wireless sensor networks," in Proceedings of the IEEE, vol. 98, no. 11, pp. 1903-1917, 2010. 